\title{
Toward a Unifying Perspective on Economic Complexity: The Contribution of Chinese Econophysics Community
}

\author{
Christophe Schinckus \\ Department of Economics and Finance, RMIT University Vietnam, Ho Chi Minh, Vietnam \\ Email: christophe.schinckus@rmit.edu.vn
}

How to cite this paper: Schinckus, C. (2018) Toward a Unifying Perspective on Economic Complexity: The Contribution of Chinese Econophysics Community. Theoretical Economics Letters, 8, 609-625. https://doi.org/10.4236/tel.2018.83042

Received: December 21, 2017

Accepted: February 23, 2018

Published: February 26, 2018

Copyright $\odot 2018$ by author and Scientific Research Publishing Inc. This work is licensed under the Creative Commons Attribution International License (CC BY 4.0).

http://creativecommons.org/licenses/by/4.0/

\begin{abstract}
Recent years have witnessed a series of economic problems and financial crises, the recent subprime debacle being one of the worst. All these events called traditional economics into question and new perspectives have been developed to take into account the complexity of economic systems. This essay aims at contributing to a better understanding of a new trend labeled econophysics through two points: 1) a discussion on the conceptual links between statistical econophysics and agent-based econophysics; and 2) an overview of works dealing with these links that interestingly, emphasize the importance of econophysics in China.
\end{abstract}

\section{Keywords}

Econophysics, Statistical Physics, Economics, Modelling of Complexity

\section{The Necessity of a Scientific Revolution}

Recent years have witnessed a series of economic problems and financial crises, the recent sub-prime debacle being one of the worst. All these events called traditional approach in economics into question and new perspectives have been developed in the literature in order to take into account the complexity of economic systems. Economics enters in a period of reflection and several scholars (Bouchaud, Farmer, Foley) wrote that "economy needs a scientific revolution" [1]. Several paths have been investigated, econophysics being one of the most important ones. Interestingly, the label econophysics often refers to a diversity of works from statistical physics to agent-based modelling [2] and the question is whether these approaches are compatible. How can such combination contribute to the scientific revolution evoked earlier? The article published in Nature by 
Bouchaud promoted the necessity to develop a new theoretical perspective in economics that is inspired from an "agent-based econophysics". However, this claim requires a clarification of the links existing between econophysics (statistical physics applied to economics) and the agent-based modelling. This essay aims at contributing to a better understanding of this new trend labeled econophysics through two points: 1) a discussion on the conceptual links between statistical econophysics and agent-based econophysics; and 2) an overview of works dealing with these links that interestingly, emphasize the importance of econophysics in China. The literature dealing with econophysics is growing and fragmented, the objective of this essay is to offer a conceptual map of the existing works by discussing the potential links between the different categories of works.

\section{Two Fields Dealing with Economic Complexity}

\subsection{Statistical Physics}

The influence of physics on financial economics is nothing new. A number of writers have studied the "physical attraction" exerted by economists on physics [3] [4]. For three decades, physicists have been going beyond the boundaries of their discipline, using their methods to study various problems thrown up by social sciences. This movement was initiated in the 1970s, when certain physicists began publishing articles devoted to the study of social phenomena. While some authors extended what is called "catastrophe theory" to social sciences, others created a new field labeled "sociophysics" [5]. The number of physicists publishing papers devoted to the analysis of social phenomena and the number of themes studied is increasing, examples being the formation of social groups [6], social mimetism [7], industrial strikes [8], democratic structures [9], and elections [10]. In the 1990s physicists turned their attention to economics, and particularly financial economics, giving rise to econophysics. Although the movement's official birth announcement came in a 1996 article by Stanley [11], econophysics was at that time still a young, ill-defined current. Mantegna and Stanley [12] defined econophysics as "a quantitative approach using ideas, models, conceptual and computational methods of physics". This definition seemed to gain ground as a compromise, and is found in a number of books and articles [13] [14] [15].

Econophysics comes from statistical physics and it is often associated to what we call "stylized facts" in the economic literature. These stylized facts mainly refer to "empirical facts that arose in statistical studies of financial (or economic) time series and that seem to be persistent across various time periods, places, markets, assets etc" [16]. Because this kind of econophysics is mainly based on a time analysis of financial or economic phenomena, it requires a lot of past data on prices, volumes, or transactions from which models describe the fat-tailed empirical distributions of returns, the absence of auto-correlation of returns or the volatility clustering. As Chakraborti et al. [17] explained, the notion of time is sometimes redefined by econophysicists since we find some works using ca- 
lendar time, event time (where time unit is based on submitted order on the market), transaction time (where time unit is based on the occurrence of a transaction) and tick time (time unit is based the prices changes). For econophysicists, economic systems are composed of multiple particles (agents) interacting in such a way as to generate the macro-properties for systems [14]. These macro-properties can be characterized in terms of statistical regularities. Econophysics is founded on the belief in the universality of the statistical properties i.e. the fact that they reappear across many and diverse phenomena [18]. In this perspective each micro-state appears to be indeterminate and econophysicists try to extract macro statistical regularities that are seen as emerging properties. These properties appear then as phenomenological regularities which refers to what Holland [19] called "macro-laws" and which are associated with the notion of "universal laws" in econophysics [20]. In the econophysical literature, we can find two ways of dealing with these emerging macro-laws: while some econophysicists try to describe these regularities directly from the evolution of these systems [18], other econophysicists rather study these systems by reproducing artificially these macro-regularities [21]. As this article explains it, these two perspective actually define the methodological scope of econophysics since the first category of works implements statistical perspective (statistical econophysics) whereas the second use an agent-based methods (agent-based econophysics $)^{1}$.

Roughly speaking, statistical econophysics can be defined as an area of knowledge that deals with the phenomenological characterization of statistical patterns that macroscopically describe the dynamics of complex economic systems. In this approach, the notion of emergence is often associated with statistical regularity that is observed in a very high number of past micro interactions. In terms of output (results), the accumulation of observations allows econophysicists to observe a specific statistical regularity, which often takes the form of a power law. Such approach has been well illustrated by Buchanan [22] as follows (Figure 1).

More precisely, the way to produce the output consists of visually checking on a simple histogram that the frequency distribution of the quantity of $\mathrm{x}$ appears as a straight line when plotted on double logarithmic axes. If a distribution falls approximately on a straight [line, then one can consider that the distribution follows a power law, with a scaling parameter a given by the absolute slope of the straight line. Such visual investigation has guided econophysicists' empirical research (Mantegna and Stanley, 1999; Jovanovic and Schinckus [23] and can be illustrated with Figure 2.

This kind of visual relationship has been observed in many financial and economic phenomena. The reasons and the way econophysicists use the log-log system was explained in detail earlier. Statistical econophysicists tend to see this linearity in a large collection of empirical observations and, according to them,

${ }^{1}$ See Schinckus [2] for further detailed review of these works. 


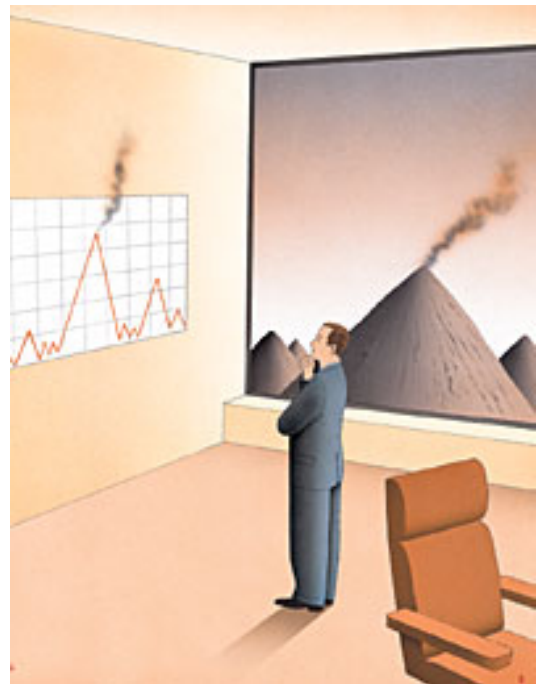

Figure 1. Source buchanan [22].

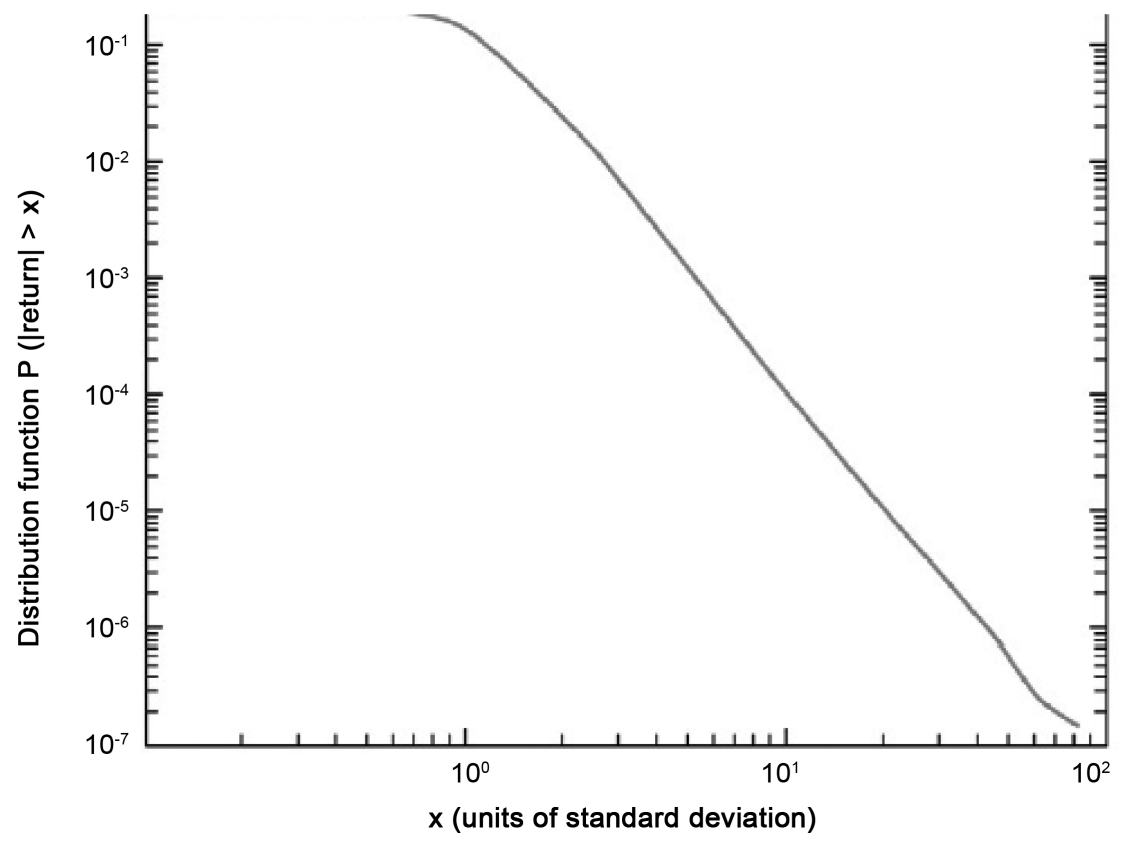

Figure 2. Empirical cumulative distribution of the absolute values of the normalized 15-min returns of the 1000 largest companies in the Trades and Quotes database for the 2-year period 1994-1995 (12 million observations)—-source: Gabaix [24].

this repetition is not due to a(n) (un)happy coincidence; rather it stresses the phenomenological universality of power laws that emerge in different contexts. This idea of universality has supported the claim, according to which methods and models from statistical physics could be applied outside physics [12] [25].

\subsection{Agent-Based Econophysics}

Agent-based modeling is based on computerized simulations of a large number of decision-makers which can interact through specified procedures. In line with 
the economic mainstream, the agent-based method focuses on the mathematical modelling of atomistic agents but, in opposition to the former, the latter does not necessary use the representative agent methodology [26]. Economic mainstream uses a methodological individualism focusing on personal characteristics of homogeneous individuals and analyzing their behaviours in terms of utility function, risk aversion, and so on. In this perspective, economists base their reasoning on the rationality of individuals maximizing a particular utility function. This condition of rationality is not necessary in agent-based models. Agents are seen as interacting components whose behaviours create different complex structures. Agents are then considered as heterogeneous and "they may differ in myriad ways genetically, culturally, by social networks, by preferences etc." [27]. The specificity of the agent-based modelling refers to the fact that this approach takes of human adaptation and learning abilities into account.

Agent-based method refers to what Arthur and al. [28] called the "small tent complexity" in which interacting heterogeneous agents create a complex hierarchical organization in a no-controlled system. This approach appeared in the 1990s as a new tool for empirical research in a lot of fields such as economics [29], voting behaviors [30], military tactics [31], organizational behaviors [32], epidemics [33], traffic congestion patterns [33], etc. The today literature about agent-based models is huge and published in several disciplines. In this perspective, agent-based models can be looked on as an interdisciplinary approach [27] and it refers to so many fields that it is not possible to number them in this paper whose objective is to focus on agent-based models and its links with econophysics where agent-based approach has also been extended.

Agent-based econophysics and statistical econophysics have common foundations since they describe socioeconomic systems as complex systems suggesting the unavoidable result of bringing together numerous components in a nonsimple manner. Moreover, these two approaches avoid arbitrary assumptions and they based their methodology on empirical verifications. However, some differences exist. The main distinction between these two fields refers to the way of modeling agents: while agent-based econophysics deals with microscopic models applied to heterogeneous and learning agents; statistical econophysics rather uses "zero-intelligence agents" (with no learning abilities) whose interactions are random. This distinction between these two subfields of econophysics has been suggested by Bouchaud [34] and evoked by Chakraborti et al. [17] who wrote an econophysics review through two excellent companion papers dedicated to the foundations of these two sub-fields. In opposition to economics or agent-based econophysics, statistical econophysics considers that only the macro-level of the system can be observed and analyzed. There is no modeling of the rational or/and individual behavior like in economic mainstream or in agent-based econophysics. Statistical econophysics rather uses a zero intelligence agents in line with the definition given by Gode and Sunder [35] where an agent who "has not intelligence, does not seek or maximize profits and does not observe, remember 
or learn". The agents' behavior is random and the result is mathematically analogous to a reaction of diffusion model in physics. While a lot of works [12] [36] in statistical econophysics do not even mention the agent-based approach at all, others works try to explain statistical regularities by using random particles (characterized by a zero-intelligence agent). Bak et al. [37], for example, provided a model in which orders were particles moving along a price line, and whose random collisions were seen as transactions (see also Famer and al [20], for the same kind of model).

These works can be methodologically characterized by a non-economic agent-based approach since non-economic assumptions are initially made/used for the calibration of micro interactions. Actually, econophysicists define algorithmic rules that generate micro interactions in terms of "physically plausible" events, implying that agents and their interactions are described with notions such as potential states (deposition, cancellation, annihilation, etc.), thermal features (heat release rate, ignition point, etc.) or magnetic dimensions (magnetic permeability, excitation). In other words, the input in such modelling is a pre-defined set of micro interactions that are physically plausible/meaningful. By applying these existing models to describe economic phenomena, econophysicists implicitly assume a kind of physicality since they consider them a social reality that can be explained in physical terms. Indeed, by using physical concepts to deal with economic/social reality, econophysicists don't deny that the world contains non-physical elements, such as items of a biological, psychological, moral or social nature, but, as Stoljar [38] explained, "they insist nevertheless that at the end of the day such items are either physical or supervene the physical" [38]. In a sense, econophysicists use this "physically plausible dimension of micro interactions" as an analogy for economic relations. This way of modelling is far from the economic-incentives-based models developed by economists. Consequently, there is no link with usual economic knowledge, which explains why this kind of agent-based econophysics is largely ignored by economists (who instead implement an economic incentives-led agent-based modelling). This is not economic calibration; in econophysics, modelling can be described by the following schema (Figure 3).

In contrast with the bottom-up agent-based approach, initial assumptions (inputs) are formulated by integrating information from a particular macro pattern observed in the past evolution of the complex system. The following diagram summarizes the modelling process of this category of works (Figure 4).

As previously evoked, the emergence of econophysics is directly associated with the identification of statistical regularities in complex economic/financial dynamics. When the statistical approach is combined with agent-based modelling, the analysis begins with the phenomenological observation of a statistical regularity in a particular economic phenomenon. Afterwards, conditions are derived from the observed macro pattern to calibrate the micro interactions of individual market participants. These micro interactions will then be algorithmically generated with the hope of quantitatively reproducing the initial macro pattern. 


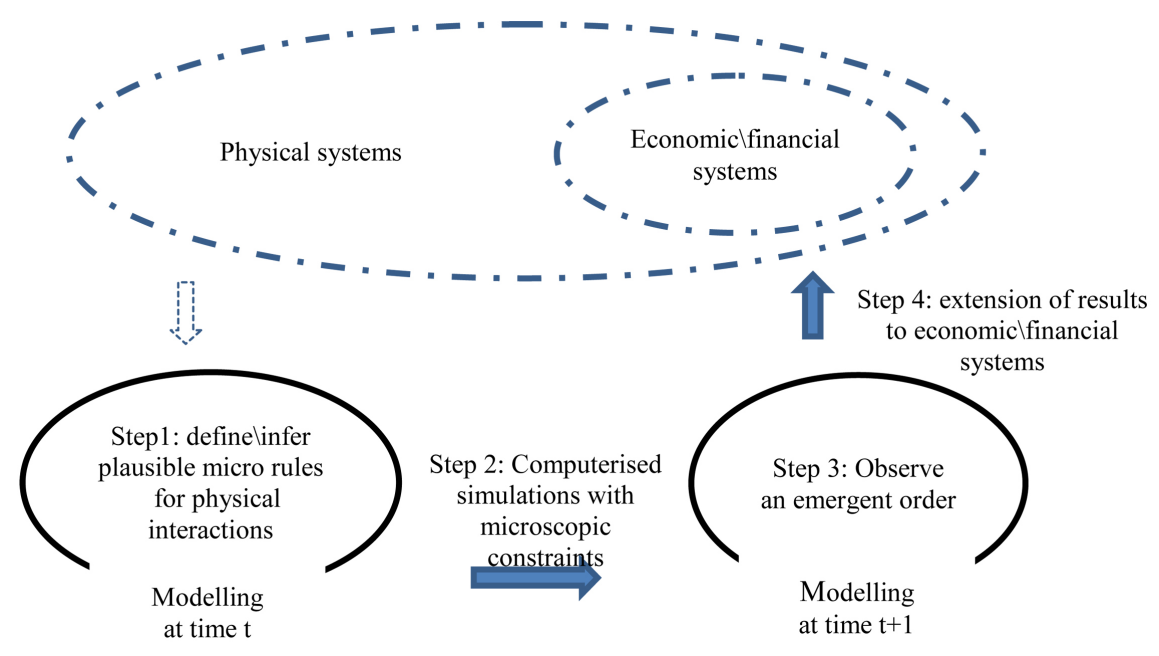

Figure 3. Modelling process for this application of agent-based modeling. Source: Schinckus [2].

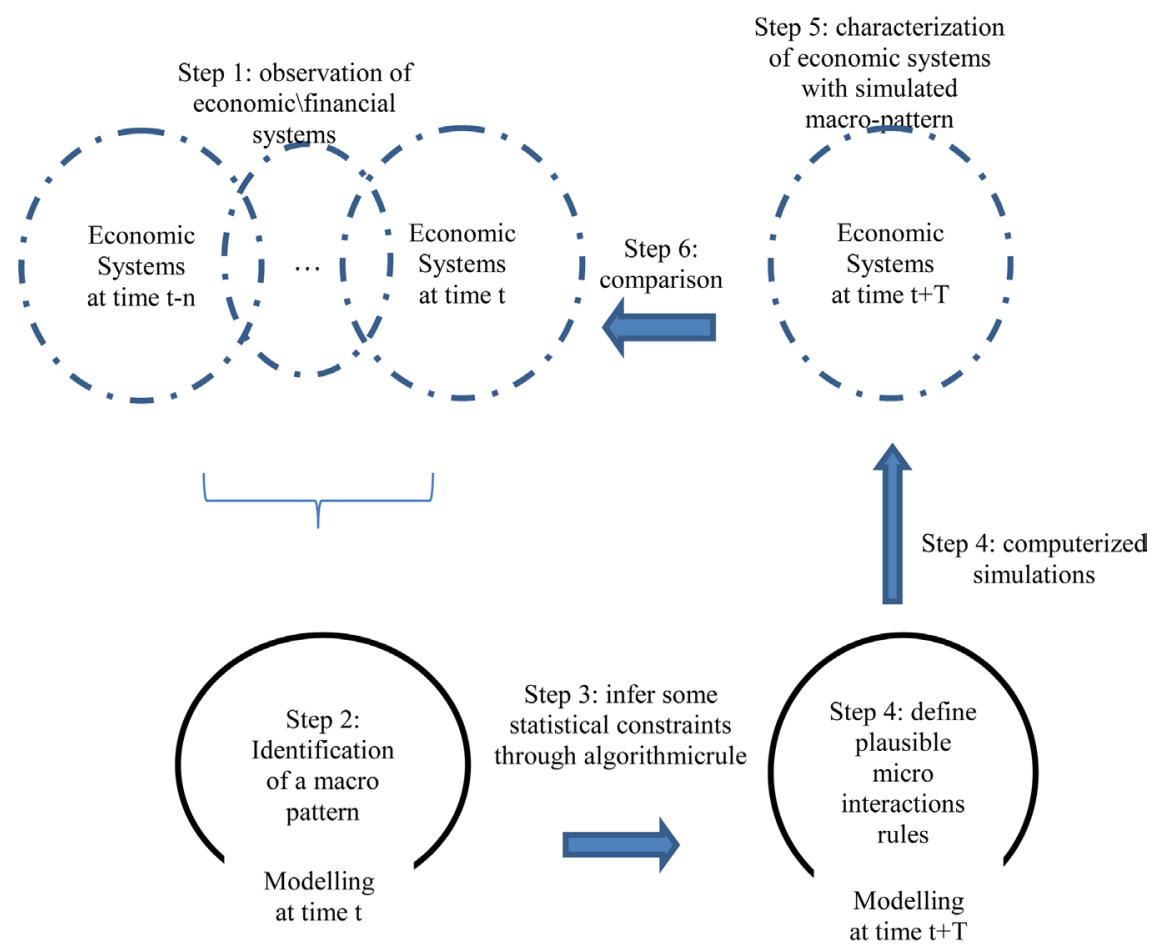

Figure 4. Modelling process of econophysics agent-based modeling. Source: Schinckus [2].

\section{Unifying Perspective on Economic Complexity}

Financial systems consist of a large number of components in which random interactions generate observable properties such as power laws, for example. While economics and agent-based econophysics share a microscopic methodology, statistical econophysics is rather founded on a macro-approach in which atoms do not learn implying the fact that all "market components" (including traders, speculators, and hedgers) obey statistical properties. In this perspective, statistic- 
al econophysicists avoid the difficult task of theorizing about the individual psychology (or rationality) of investors [39]. Statistical econophysics also avoid the difficult process of calibration of the agent-based models (which appears to be the main drawback of agent-based approach, [40]). Statistical econophysicists do not care about rational agent theory or the personal characteristics (utility and so on) of individuals. Knowledge developed by this kind of models mainly results from analysis of past data that authors try to explain through complex statistical processes. In a sense, the main objective of statistical econophysics is to describe the past financial and economic data through models. In line with what Stephan [41] called "synchronic emergent phenomenon", econophysics macro-laws refer to past phenomena (historical analysis of empirical data) in which micro-entities cannot learn or adapt (zero-intelligent agents) themselves across time.

Agent-based econophysics is rather founded on heterogeneous agent-based modelling. Agent-based econophysics has mainly developed models of orderdriven markets (related to microstructure), game theory models (by redefining the minority problems and related problems) or models using kinetic theory [17]. We can also find works [21] [42] which studied the emergence of money through studying the dynamics of exchange in a system composed of many learning agents. While statistical econophysics uses historical data with (eventually) a zero-intelligence agent, agent-based econophysics rather produces data through a complex modeling of self-evolving systems (with learning agents). These learning abilities of agents lead to a no "final equilibrium" and this kind of situation can then be treated with specific analytical tools used in physics such as Fokker-Planck equations, Langevin equation etc. In this perspective, the emergence enhanced by agent-based econophysics refers to a "diachronic emergence" [41], in which all emergent phenomena occur across time by means of complex adaptation of learning agents. The main objective of the agents based econophysics is the "reproduction of the phenomenon" and not only the statistical description. This importance of reproducibility of data can be observed through all the literature dedicated to agents-based models [17] [40].

Is agent-based modeling compatible with the scientific revolution evoked in the Bouchaud's paper in Nature? The answer is yes. Although econophysics and agent-based approach have different history [43], they appear to be complementary fields. Indeed, econophysics offers analytical tools to describe and characterize the macro-statistical regularities in the evolution of complex systems while agent-based modelling provides a framework reproducing these statistical regularities by giving them behavioural and micro-foundations. The complementary nature between these two fields refers then to their different ways of dealing with complexity. A strictly statistical approach gives us a lot quantitative information about macro-outcomes observed in real complex economic systems. Econophysics contributed to an increasing awareness for statistical properties such as power-law tails of distributions of returns, temporal scaling of volatility, fractal- 
ity of time etc). Econophysicists provided interesting analytical tools in order to deal with these complex concepts. The main drawback of econophysics is the lack of knowledge of economic theory and these works are often driven more by mathematical convenience than economic meaning [44]. Agent-based modelling offers a way to describe economic systems as a complex system as Keynes attempted to do i.e. by taking of human adaptation and learning into account [28]. The heterogeneity of behaviors combined with the learning abilities of agents imply the possibility to give an economic meaning to the micro-interactions generating macro-properties. Agent-based approach does not directly observe the real complex systems, it rather attempts to reproduce them in order to find the same statistical regularities observed in the real complex systems. Because this field finds the same regularities then those observed by econophysicists; and because it provides micro-foundations (and then potentially behavioral explanations) to the statistical regularities that emerge at the macro-level of the systems, the agent-based approach can be considered as a complementary field with a strictly statistical approach. Econophysics developed analytical tools improving the description of macro-properties observed in the economic complex systems and agent-based method provided many contributions for the understanding of complex micro-interactions that cause these macro-properties. As Farmer and Foley [20] emphasized it, "agent-based method provides an unprecedented understanding of the emergent properties of interacting parts in complex systems circumstances where intuition fails".

This historical review allowed me to present the two major computational approaches (agent-based modelling and statistical invariance) for describing the complicated process associated with a dynamic complexity. Although these two computational approaches both emerged in the same institution, one could wonder what these two computational approaches have in common. As Waldrop [45] explained, a lot of scientists working on complexity in the 1980s acknowledged that, at first sight, "Bak's critical state [statistical perspective] didn't seem to have anything to do with life or computation [usually modelled with an agent-based approach]". However, it is worth mentioning that these two approaches share the same foundations since they study complex systems through the dynamics of numerous components interacting in a non-simple manner. Moreover, these two computational techniques use a methodology based on empirical verifications. Some scientists (Langston, Kauffman) affiliated to the Santa Fe Institute were fascinated by the potential connection between these two computational approaches of dynamic complexity: "Langston had done the hard work of making the idea rigorous and precise" [45]. More precisely, Langston [46] [47] [48] proposed a formal connection between the dynamics of critical states and the one observed in computerized computation, "computation may emerge spontaneously and come to dominate the dynamics of physical systems when those systems are at or near a transition between their solid and fluid phases, especially in the vicinity of a second-order or critical transition" [48]. 
In other words, "we observe surprising similarities between the behaviors of computations and systems near phase [critical] transitions, finding analogs of complexity classes" (Langston, 1990, p. 12). These works opened the door to "unificationist dreams" based on the potential creation of "the science of all sciences" [49] as or a "big science of complexity" [50] [51]. Although that idea of big science influenced the methodological evolution of econophysics, this doctoral research will not present complexity as a "big science" because the prescientific status of a potential unified science of complexity combined to the great number of involved disciplines generate so large diversity of theories that models and explanations related to complexity necessary required a pluralist perspective [52].

Economics needs a scientific revolution that demands a multi-disciplinary collaboration between economists, computers scientists, psychologists and physicists. One potential direction for this revolution is the development of an agent-based econophysics. This multi-disciplinary field can indeed help scholars to better understand the complexity of economic systems but it can also produce very useful tools for policy-makers such as, for example, the work of Pickhardt and Seibold [53] which gave a realistic description of that income tax evasion dynamics by modelling it through an "agent-based econophysics model" based on Ising model of ferromagnetism. The following section emphasizes the contributions of scientific the Chinese community to econophysics.

\section{Econophysics in China: An Important Community!}

The most popular area of knowledge investigated by Chinese econophysicists refers to the identification of macro-patterns in economic $\backslash$ financial data. Nowadays, China is playing a key role in the development of econophysics as witnessed the recent (May 2017) international conference organized by the Research Center for Econophysics at the East China University of Science and Technology (http://ice2017.csp.escience.cn/dct/page/65579) in Shanghai. This center explicitly promotes research in econophysics and its members are well-established in the international community of econophysicists. Members of this research center work on diverse aspects of the field such as the dynamics of short-term returns in ultra-high-frequency on the Shenzen stock exchange [54] or the description of the minutes records of the Hang Seng index [55] This group is also well-known for its research on the identification of time-dependent patterns in the dynamics of financial returns [56] [57] [58].

Several Chinese universities developed a strong expertise in econophysics. Beijing Normal University, for instance, has a strong group of econophysicists working on the characterization of macro-patterns on Chinese stock markets [59] and wealth distribution in China [57] [60]. This university also developed international collaborations with key econophysicists such as Prof. Bertrand Roehner who became visiting professor in this institution in 2009.

Peking University also host several scholars working on econophysics. Zhang 
[61] worked on the statistical description of the Chinese stock markets while Ahn et al. [62] deal with the infinite variance in option pricing. Beihang University has a small group of econophysicists led by $\mathrm{Ch}$. Gou who worked on simulated financial markets (Gou [50]) and volatility of financial prices (Gou and al. [63]). Fudan University regularly publishes articles related to econophysics thanks to P. Chen who study financial crisis through the lens of transition phase.

This quick overview only mentioned some of the key universities involved in econophysics research; however, it is worth mentioning the existence of several Chinese scholars working on econophysics without having a particular institutional umbrella. For instance, W. Zhang (Tianjin University) who regularly publishes articles in econophysics [64] [65] but other econophysicists can also be [66] [67] [68].

Schinckus [69] explained that the literature dealing with agent-based models coming from physics but applied in economics can be decomposed into two categories: on one hand, we have a research characterizing the emergence of specific macro-properties without using a pre-defined macro-pattern, and on the other hand, one can find works whose objective is to reproduce an existing (pre-defined) macro-statistical pattern. The majority of papers dealing with $\mathrm{ABM}$ in econophysics concern research for which micro-interactions are considered as an input and the emerging macro-result is looked on as an output of the process. Agents are then considered as interacting particles whose adaptive behaviours create different structures (such as molecules, cells, crystals, etc). Chinese econophysicists also contributed significantly to this specific literature. Li [70] for instance offered an agent modelling based explanation for some financial characteristics of assets returns while Zheng et al. [71] rather focused on the agent-based modelling of herding behaviour combined with phase-transition analysis to describe financial dynamics. Wang [72] developed an evolutionary game theoretical model to describe the dynamics of wealth distributions in China. Interestingly, the agent-based econophysics appears to be a thematic research at the Zhejing University where several scholars directly contributed to this field. Zheng et al. [73], for instance, proposed a multi-agent based model to simulate the microscopic interactions and the dynamic evolution of financial markets. Zhou et al. [67] developed a bottom-up model to explain the emergence of auto-correlation in stock returns whereas Chen et al. [74] [75] characterized the herding behaviours in terms of multi-agent based modelling. Finally, Chen et al. [76] promoted the use of agent-based modelling to characterize the complexity of financial systems at the era of big data.

The last category of works dealing with $\mathrm{ABM}$ of economic systems refers to a research whose objective is to reproduce existing statistical data. In opposition to the previous categories of works, authors involved in this area of knowledge usually refer to existing empirical studies which have previously shown the persistence of a specific statistical pattern in economic data. This observation of a macro-statistical pattern is associated with the identification of a discernible and 
noteworthy phenomenon [77]. Once this phenomenon is identified, the objective is to use its statistical macro-properties as an input for the calibration of micro-interactions which are then supposed to generate the macro-patterns initially observed. Feng et al. [[78], p. 8388] explained it, "the interaction strength between agents need to be adjusted with agent population size or interaction structure to sustain fat tails in return distributions [i.e. macro-law]"2. In their study, Feng et al. [78] derived agents' micro-behaviours from scaling properties observed for the financial returns' distributions. In the same vein, Chen [79] proposed micro-foundations to the evolution of financial prices by inducing micro-patterns from the statistical behaviours of returns volatility. Ren et al. [80] suggested the same kind of top-down methodology to explain how financial traders individually act with trading volumes while $\mathrm{Hu}$ et al. [81] used this approach to give micro-foundations to the wealth distributions in China.

\section{Conclusions}

In a challenging context for economics, it is important to understand which the potential alternative frameworks are to better capture the complexity of economic phenomena. This essay offers a discussion on the methodological diversification observed in econophysics by clarifying the links between statistical econophysics and agent-based econophysics. This link has then been presented further by emphasizing the key contributions of the Chinese econophysics to the field.

This article showed a diversity of methodologies in the new field called econophysics. In a sense, this diversity is challenging because it questions the coherence of the field itself. Authors often developed their approach by staying behind their disciplinary frontiers and by presenting their methodological approach as completely new when sometimes, it is not [15]. In other words, the major challenge for the field is to become more transdisciplinary by integrating some concepts that can make sense for all scientific communities involved in the development of the field (economists, physicists and computer scientists).

\section{References}

[1] Bouchaud, J.-P. (2009) Economics Needs a Scientific Revolution. Nature, 455, 1181. https://doi.org/10.1038/4551181a

[2] Schinckus, C. (2016) 1996-2016: Two Decades of Econophysics: Between Methodological Diversification and Conceptual Coherence. Special Topics Issue Discussion \& Debate: Can Economics Be a Physical Science? The European Physical Journal, 225, 3299-3311.

[3] Mirowski, P. (1989) More Heat than Light: Economics as Social Physics, Physics as Nature's Economics. Cambridge University Press, Cambridge. https://doi.org/10.1017/CBO9780511559990

${ }^{2}$ It is worth emphasising that econophysicists keep a physical vocabulary in their definition of the interaction rules since they talked about "interaction strength" or "interaction structure" while economists rather use words "interactions" and "network". 
[4] Schabas, M. (1990) A World Ruled by Number: William Stanley Jevons and the Rise of Mathematical Economics. Princeton University Press, Princeton, N.J. https://doi.org/10.1515/9781400861514

[5] Galam, S., Yuval, G., et al. (1982) Sociophysics: A Mean Behavior Model for the Process of Strike. The Journal of Mathematical Sociology, 9, 1-13. https://doi.org/10.1080/0022250X.1982.9989929

[6] Weidlich, W. (1971) The Statistical Description of Polarization Phenomena in Society. British Journal of Mathematical and Statistical Psychology, 1, 251-266. https://doi.org/10.1111/j.2044-8317.1971.tb00470.x

[7] Callen, E. and Shapiro, D. (1974) A Theory of Social Imitation. Physics Today, 12, 23-28. https://doi.org/10.1063/1.3128690

[8] Galam, S. (1986) Majority Rule, Hierarchical Structures and Democratic Totalitarism: A Statistical Approach. Journal of Mathematical Psychology, 30, 426-434. https://doi.org/10.1016/0022-2496(86)90019-2

[9] Galam, S. (2004) Sociophysics: A Personal Testimony. Physica A, 336, 49-55. https://doi.org/10.1016/j.physa.2004.01.009

[10] Ferreira, P. and Dionisio, A. (2008) Voters' Dissatisfaction, Abstention and Entropy: Analysis in European Countries. CEFAGE-UE Working Papers 2008-11, University of Evora, CEFAGE-UE.

[11] Stanley, H., Afanasyev, V., Aamaral, L.A.N., Buldyrev, S.V., Goldberger, A.L., Havlin, S., Leschhorn, H., Maass, P., Mantegna, R., Peng, C.-K., Prince, P.A., Salinger, M.A., Stanley, M.H.R. and Viswanathan, G.M. (1996) Anomalous Fluctuations in the Dynamics of Complex Systems: From DNA and Physiology to Econophysics. Physica A, 224, 302-321. https://doi.org/10.1016/0378-4371(95)00409-2

[12] Mantegna, R. and Stanley, E. (1999) An Introduction to Econophysics. Cambridge University Press, New York. https://doi.org/10.1017/CBO9780511755767

[13] Wang, Y., Wu, J. and Zengru, D. (2004) Physics of Econophysics. Working Paper of Beijing Normal University, No. 1025.

[14] Rickles, D. (2007) Econophysics for Philosophers. Studies in History and Philosophy of Modern Physics, 38, 948-978. https://doi.org/10.1016/j.shpsb.2007.01.003

[15] Lux, T. (2009) Applications of Statistical Physics in Finance and Economics. In: Rosser Jr., J.B., Ed., Handbook of Complexity Research, Edward Elgar, Cheltenham. https://doi.org/10.4337/9781781952665.00017

[16] Chakraborti, A., Muni Toke, I., Patriarca, M. and Abergel, F. (2011) Econophysics Review: I. Empirical Facts. Quantitative Finance, 11, 991-1012. https://doi.org/10.1080/14697688.2010.539248

[17] Chakraborti, A., Muni Toke, I., Patriarca, M. and Abergel, F. (2011) Econophysics Review: I. Agent-Based Models. Quantitative Finance, 11, 1013-1041. https://doi.org/10.1080/14697688.2010.539249

[18] McCauley, J. (2004) Dynamics of Markets: Econophysics and Finance. Cambridge University Press, Cambridge. https://doi.org/10.1017/CBO9780511606588

[19] Holland, J. (1999) Emergence: From Chaos to Order. Perseus Books Editions, New York.

[20] Roehner, B.M. (2002) Patterns of Speculation: A Study in Observational Econophysics. Cambridge University Press, Cambridge. https://doi.org/10.1017/CBO9780511613494

[21] Shinohara, S. and Gunji, Y. (2001) Emergence and Collapse of Money through Reciprocity. Applied Mathematics and Computation, 117, 131-150. 
https://doi.org/10.1016/S0096-3003(99)00169-1

[22] Buchanan, M. (2013) What Has Econophysics Ever Done for Us? Nature Physics, 9, 317.

[23] Jovanovic, F. and Schinckus, C. (2017) Econophysics and Financial Economics: An Emerging Dialogue. Oxford University Press, New York. https://doi.org/10.1093/acprof:oso/9780190205034.001.0001

[24] Gabaix, X. (2009) Power Laws in Economics and Finance. Annual Review of Economics, 1, 255-293. https://doi.org/10.1146/annurev.economics.050708.142940

[25] McCauley, J. (2006) Response to "Worrying Trends in Econophysics". Physica A: Statistical Mechanics and Its Applications, 371, 601-609. https://doi.org/10.1016/j.physa.2006.05.043

[26] Kirman, A. (1992) Whom or What Does the Representative Individual Represent. Journal of Economic Perspectives, 6, 117-136. https://doi.org/10.1257/jep.6.2.117

[27] Epstein, J.M. (2006) Generative Social Science: Studies in Agent-Based Computational Modeling. Princeton University Press, Princeton.

[28] Arthur, W.B., Durlauf, S.N. and Lane, D.A. (1997) Introduction. In: Arthur, W.B., Durlauf, S.N. and Lane, D.A., Eds., The Economy as an Evolving Complex System II, Proceedings Volume XVII, Addison-Wesley, Reading, MA, 1-14.

[29] Axtell, R. (1999) The Emergence of Firms in a Population of Agents: Local Increasing Returns, Unstable Nash Equilibria, and Power Law Size Distributions. Santa Fe Institute Working Paper 99-03-019.

[30] Lindgren, K. and Nordahl, M.G. (1994) Cooperation and Community Structure in Artificial Ecosystems. Artificial Life, 1, 15-37. https://doi.org/10.1162/artl.1993.1.1_2.15

[31] Ilachinski, A. (1997) Irreducible Semi-Autonomous Adaptive Combat (ISAAC): An Artificial-Life Approach to Land Warfare. Center for Naval Analyses Research Memorandum, CRM 97-61.10. https://doi.org/10.21236/ADA362371

[32] Prietula, M., Carley, K. and Gasser, L. (1998) Simulating Organizations: Computational Models of Institutions and Groups. The MIT Press, Cambridge, MA.

[33] Epstein, J.M and Axtell, R. (1996) Growing Artificial Societies: Social Science form Bottom Up. MIT Press, Cambridge.

[34] Bouchaud, J.-P. (2002) Introduction to Statistical Physics. Physica A: Statistical Mechanics and Its Applications, 313, 338-351. https://doi.org/10.1016/S0378-4371(02)01039-7

[35] Gode, D. and Sunder, S. (1993) Allocative Efficiency of Markets with Zero-Intelligence Traders: Market as a Partial Substitute for Individual Rationality. Journal of Political Economic, 101, 119-137. https://doi.org/10.1086/261868

[36] Huang, Z. and Salomon, S. (2001) Power, Levy, Exponential and Gaussian-Like Regimes in Autocatalytic Financial Systems. The European Physical Journal B-Condensed Matter and Complex Systems, 20, 601-607. https://doi.org/10.1007/PL00011114

[37] Bak, P., Paczuski, M. and Shubik, M. (1997) Price Variations in a Stock Market with Many Agents. Physica A: Statistical Mechanics and Its Applications, 246, 430-453. https://doi.org/10.1016/S0378-4371(97)00401-9

[38] Stoljar, D. (2010) Physicalism. Routledge, New York.

[39] Brandouy, O. (2005) Complexité et phénomènes critiques en finance. In: Bourghelle, D., Brandouy, O., Gillet, R. and Orléan, A., Eds, Croyances, Représentations 
Collectives et Conventions en Finance, Economica, Paris.

[40] LeBaron, B. (2006) Agent-Based Computational Finance. In: Tesfatsion, L. and Judd, K.L., Eds., Handbook of Computational Economics, Volume 2: Agent-Based Computational Economics, North-Holland, Amsterdam, 1187-1233.

[41] Stephan, A. (2002) Emergence. In: Nadel, Ed., Encyclopedia of Cognitive Science Vol. 1, Macmillan, London, 1108-1115.

[42] Donangelo, R. and Sneppen, K. (2000) Self-Organization of Value and Demand. Physica A: Statistical Mechanics and Its Applications, 276, 572-580. https://doi.org/10.1016/S0378-4371(99)00473-2

[43] Schinckus C. (2017) When Physics Became Undisciplined: An Essay on Econophysics. Ph.D. Dissertation, University of Cambridge, Cambridge.

[44] Alfarano, S., Lux, T. and Wagner, F. (2005) Estimation of Agent-Based Models: The Case of an Asymmetric Herding Model. Computational Economics, 26, 19-49. https://doi.org/10.1007/s10614-005-6415-1

[45] Waldrop, M. (1992) Complexity: The Emerging Science at the Edge of Order and Chaos. Simon \& Schuster Editions, New York.

[46] Langton, C. (1986) Studying Artificial Life with Cellular Automata. Physica D: Nonlinear Phenomena, 22, 120-149. https://doi.org/10.1016/0167-2789(86)90237-X

[47] Langton, C. (1988) Artificial Life: Proceedings of an Interdisciplinary Workshop on the Synthesis and Simulation of Living Systems. Addison-Wesley, Reading, MA.

[48] Langton, C. (1990) Computation at the Edge of Chaos. Physica D: Nonlinear Phenomena, 42, 125-135. https://doi.org/10.1016/0167-2789(90)90064-V

[49] Johnson, N. (2007) Simply Complexity: A Clear Guide to Complexity Theory. Oneworld Publications, Oxford.

[50] Mitchell, M. (2009) Complexity: A Guided Tour. Oxford University Press, New York.

[51] Johnson, N., Jeffries, P. and Hui Pak, K. (2003) Financial Market Complexity. Oxford University Press, Oxford. https://doi.org/10.1093/acprof:oso/9780198526650.001.0001

[52] Mitchell, S. (2003) Biological Complexity and Integrative Pluralism. Cambridge University Press, Cambridge. https://doi.org/10.1017/CBO9780511802683

[53] Pickhardt, M. and Seibold, G. (2011) Income Tax Evasion Dynamics: Evidence from an Agent-Based Econophysics Model. Working Paper of the University of Cottbus.

[54] Zhou, J., Gu, G.F., Jiang, Z.Q., Xiong, X., Zhang, W. and Zhou, W.X. (2016) Computational Experiments Successfully Predict the Emergence of Auto-Correlations in Ultra-High-Frequency Stock Returns. Computational Economics. (In Press)

[55] Wang, B.H. and Hui, P.M. (2001) The Distribution and Scaling of Fluctuations for Hang Seng Index in Hong Kong Stock Market. European Physical Journal B, 20, 573-579.

[56] Jiang, X.F. and Zheng, B. (2012) Anti-Correlation and Subsector Structure in Financial Systems. European Physical Journal B, 97, 48006.

[57] Jiang, X.F., Chen, T.T. and Zheng, B. (2013) Time-Reversal Asymmetry in Financial Systems. Physica A: Statistical Mechanics and Its Applications, 392, 5369-5375. https://doi.org/10.1016/j.physa.2013.07.006

[58] Shi, H.-L., Jiang, Z.-Q. and Zhou, W.-X. (2017) Time-Varying Return Predictability in the Chinese Stock Market. Reports in Advances of Physical Sciences, 1, 328-342.

[59] Li, H.G., Wang, D.H. and Chen, X.G. (2004) Job Match and Income Distributions. 
Physica A: Statistical Mechanics and Its Applications, 341, 569-574.

https://doi.org/10.1016/j.physa.2004.05.030

[60] Li, H.G. and Gao, Y. (2008) A GDP Fluctuation Model Based on Interacting Firms. Physica A: Statistical Mechanics and Its Applications, 387, 5225-5230. https://doi.org/10.1016/j.physa.2008.05.016

[61] Zhang, J.H., Chen, Q.H., Wang, Y.G, Zipf Distribution in Top Chinese Firms and an Economic Explanation. Physica A: Statistical Mechanics and Its Applications, 388, 2020-2024. https://doi.org/10.1016/j.physa.2009.01.027

[62] Ahn, K., Tarzia, D. and Muliere, P. (2012) Infinite Variance and Self-Similarity in Option Prices. Working Paper, Peking University.

[63] Gou, C.L., Shi, L.L., Wang, Y.W., Chen, D., Han, L.Y. and Piao, Y. (2010) A Security Price Volatile Trading Conditioning Model. Social Science Research Network.

[64] Zheng, B., Jiang, X.F. and Ni, P.Y. (2014) A Mini-Review on Econophysics: Comparative Study of Chinese and Western Financial Markets. Chinese Physics B, 23, 078903. https://doi.org/10.1088/1674-1056/23/7/078903

[65] Qiu, T., Zheng, B., Ren, F. and Trimper, S. (2006) Return Volatility Correlation in Financial Dynamics. Physical Review E, 73, 065103. https://doi.org/10.1103/PhysRevE.73.065103

[66] Gou, C.L. (2006) The Simulation of Financial Markets by Agent-Based Mix-Game Models. Journal of Artificial Societies and Social Simulations, 9, 222-230.

[67] Jiang, X.F., Chen, T.T. and Zheng, B. (2014) Structure of Local Interactions in Complex Financial Dynamics. Scientific Report, 4, Article Number: 5321. https://doi.org/10.1038/srep05321

[68] Tan, L., Zheng, B., Chen, J.J. and Jiang, X.F. (2014) How Volatilities Nonlocal in Time Affect the Price Dynamics in Complex Financial Systems. PLoS ONE, 10, e0118399.

[69] Schinckus, Ch. (2013) Econophysics, a New Step in the Evolution of Physical Sciences. Contemporary Physics, 54, 17-32.

https://doi.org/10.1080/00107514.2013.774528

[70] Li, H.G. and Rosser, J.B. (2004) Market Dynamics and Stock Price Volatility. The European Physical Journal B-Condensed Matter and Complex Systems, 39, 409-413. https://doi.org/10.1140/epjb/e2004-00206-x

[71] Zheng, B., Qiu, T. and Ren, F. (2004) Two-Phase Phenomena, Minority Games and herding models. Physical Review E, 69, 046115.

https://doi.org/10.1103/PhysRevE.69.046115

[72] Wang, W., Chen, Y. and Huang, J.P. (2009) Heterogeneous Preferences, Decision-Making Capacity, and Phase Transitions in a Complex Adaptive System. Proceedings of the National Academy of Sciences, 106, 8423-8428. https://doi.org/10.1073/pnas.0811782106

[73] Zheng, B., Jiang, X.F. and Chen, T.T. (2014) Structure of Local Interactions in Complex Financial Dynamics. Scientific Report, 4, 5321.

[74] Chen, J.J., Zheng, B. and Tan, L. (2015) Agent-Based Model with Multi-Level Herding for Complex Financial Systems. Scientific Report, 5, Article Number: 8399. https://doi.org/10.1038/srep08399

[75] Chen, J.J., Zheng, B. and Tan, L. (2013) Agent-Based Model with Asymmetric Trading and Herding for Complex Financial Systems. PLoS ONE, 8, e79531. https://doi.org/10.1371/journal.pone.0079531

[76] Chen, T., Jiang, X.F. and Zheng, T. (2013) Structure of Local Interactions in Com- 
plex. Financial Dynamics. Working Paper, Zhejiang University, Hangzhou.

[77] Wan, Y.L., Xie, W.J., Gu, G.F., Jiang, Z.Q., Chen, W., Xiong, X., Zhang, W. and Zhou, W.X. (2015) Statistical Properties and Pre-Hit Dynamics of Price Limit Hits in the Chinese Stock Markets. PLoS ONE, 10, e0120312.

https://doi.org/10.1371/journal.pone.0120312

[78] Feng, L., Li, B., Podobnik, B., Preis, T. and Stanley, H.E. (2012) Linking Agent-Based Models and Stochastic Models of Financial Markets. Proceedings of the National Academy of Sciences, 109, 8388-8393. https://doi.org/10.1073/pnas.1205013109

[79] Chen, T.T., Zheng, B., Li, Y. and Jiang, X.F. (2017) New Approaches in Agent-Based Modeling of Complex Financial Systems. Frontiers of Physics, 12, 128905. https://doi.org/10.1007/s11467-017-0661-2

[80] Ren, F., Zheng, B. and Chen, P. (2010) Modeling Interactions of Trading Volumes in Financial Dynamics. Physica A: Statistical Mechanics and Its Applications, 389, 2744-2750. https://doi.org/10.1016/j.physa.2010.02.039

[81] Hu, M.B., Jiang, R., Wu, Y.H., Wang, R.L. and Wu, Q.S. (2008) Properties of Wealth Distribution in Multi-Agent Systems of a Complex Network. Physica A: Statistical Mechanics and Its Applications, 387, 5862-5867.

https://doi.org/10.1016/j.physa.2008.06.032 